\title{
The Generalized Counting Rule and Oscillatory Scaling
}

\author{
D. Dutta, H. Gao \\ Triangle Universities Nuclear Laboratory and \\ Department of Physics, Duke University, Durham, NC 27708, USA
}

\begin{abstract}
We have studied the energy dependence of the $p p$ elastic scattering data and the pionphotoproduction data at $90^{\circ} \mathrm{c} . \mathrm{m}$. angle in light of the new generalized counting rule derived for exclusive processes. We show that by including the helicity-nonconserving amplitudes and their interference with the Landshoff amplitude, we are able to reproduce the energy dependence of all the $p p$ elastic cross-section and spin-correlation $\left(\mathrm{A}_{N N}\right)$ data available above the resonance region. The pion-photoproduction data can also be described by this approach, however, data with much finer energy spacing is needed to confirm the oscillations about the scaling behavior. This study strongly suggests an important role for helicity-nonconserving amplitudes related to quark orbital angular momentum and for the interference of these amplitudes with the Landshoff amplitude at $\mathrm{GeV}$ energies.

PACS numbers: 13.75.Cs, 24.85.+p, 25.10.+s, 25.20.-x
\end{abstract}

The transition between perturbative and nonperturbative regimes of Quantum Chromo Dynamics (QCD) is of long-standing interest in nuclear and particle physics. Exclusive processes play a central role in studies trying to map out this transition. The differential cross sections for many exclusive reactions [1] at high energies and large momentum transfers appear to obey dimensional scaling laws 2] (also called quark counting rules). In recent years, the onset of this scaling behavior has been observed at a hadron transverse momentum of $\sim 1.2(\mathrm{GeV} / \mathrm{c})$ in deuteron photo-disintegration [3, 4] and in pion photoproduction from nucleon [5]. On the other hand, these models also predict hadron helicity conservation in exclusive processes [6], and experimental data in similar energy and momentum regions tend not to agree with these helicity conservation selection rules [7]. Although contributions from non-zero parton orbital angular momenta are power suppressed, as shown by Lepage and Brodsky 8], they could break hadron helicity conservation rule [9]. Interestingly recent re-analysis of quark orbital angular momenta seems to contradict the notion of power suppression [10. Furthermore, Ref 11] argues that non-perturbative processes could still be important in some kinematic regions even at high energies. Thus the transition between the perturbative and nonperturbative regimes remains obscure and makes it essential to understand the exact mechanism governing the early onset of scaling behavior.

Towards this goal, it is important to look closely at claims of agreement between the differential cross section data and the quark counting rule prediction. Deviations from the quark counting rules have been found in exclusive reactions such as elastic proton-proton $(p p)$ scattering [12, 13]. In fact, the re-scaled $90^{\circ}$ center-ofmass $p p$ elastic scattering data, $s^{10} \frac{d \sigma}{d t}$ show substantial oscillations about the power law behavior. Oscillations are not restricted to the $p p$ elastic scattering channel; they are seen in elastic $\pi p$ fixed angle scattering 14 and hints of oscillation about the $s^{-7}$ scaling have also been reported in the recent data [5] from Jefferson Lab (JLab) on photo-pion production above the resonance region. In addition to violations of the scaling laws, spin correlations in polarized $p p$ elastic scattering also show significant deviations from perturbative QCD (pQCD) expectations 15, 16]. Several sets of arguments have been put forward to account for these deviations from scaling laws and the unexpected spin correlations. Brodsky and de Teramond 17] explain the $p p$ scattering data in terms of the opening up of the charm channel and excitation of cc̄uuduud resonant states. Alternatively the deviations are said to be an outcome of the interference between the pQCD (short distance) and the long distance Landshoff amplitude (arising from multiple independent scattering between quark pairs in different hadrons) 18]. Gluonic radiative corrections to the Landshoff amplitude give rise to an energy dependent phase [19] and thus the energy dependent oscillation. Carlson, Chachkhunashvili, and Myhrer 20] have also applied a similar interference concept to explain the $p p$ polarization data. The QCD re-scattering calculation of the deuteron photo-disintegration process by Frankfurt, Miller, Sargsian and Strikman 21] predicts that the additional energy dependence of the differential cross-section, beyond the $\frac{d \sigma}{d t} \propto s^{-11}$ scaling, arises primarily from the $n-p$ scattering in the final state. In this scenario the oscillations may arise due to QCD final state interaction. If these predictions are correct, such oscillatory behavior may be a general feature of high energy exclusive photoreactions.

Recently, a number of new developments have generated renewed interest in this topic. Zhao and Close 22 have argued that a breakdown in the locality of quarkhadron duality (dubbed as "restricted locality" of quarkhadron duality) results in oscillations around the scaling curves predicted by the counting rule. They explain that the smooth behavior of the scaling laws arise due 
to destructive interference between various intermediate resonance states in exclusive processes at high energies. However, at lower energies this cancellation due to destructive interference breaks down locally and gives rise to oscillations about the smooth behavior. On the other hand, Ji et al. 23] have derived a generalized counting rule based on a pQCD inspired model, by systematically enumerating the Fock components of a hadronic light-cone wave function. Their generalized counting rule for hard exclusive processes include parton orbital angular momentum and hadron helicity flip, thus they provide the scaling behavior of the helicity flipping amplitudes. The interference between the different helicity flip and non-flip amplitudes offers a new mechanism to explain the oscillations in the scaling cross-sections and spin correlations. The counting rule for hard exclusive processes has also been shown to arise from the correspondence between the anti-de Sitter space and the conformal field theory [24] which connects superstring theory to conformal gauge theory. Brodsky et al. 25 have used this anti-de Sitter/Conformal Field Theory correspondence or string/gauge duality to compute the hadronic light front wave functions. This yields an equivalent generalized counting rule without the use of perturbative theory. Moreover, pQCD calculations of the nucleon formfactors including quark orbital angular momentum [26, 27] and those computed from light-front hadron dynamics 25] both seem to explain the $\frac{1}{Q^{2}}$ fall-off of the proton form-factor ratio, $G_{E}^{p}\left(Q^{2}\right) / G_{M}^{p}\left(Q^{2}\right)$, measured recently at JLab in polarization transfer experiments 28].

In this letter we examine the role of the helicity flipping amplitudes in the oscillatory scaling behavior of $p p$ scattering and charged photo-pion production from nucleons and the oscillations in the spin correlations observed in polarized $p p$ scattering. We have used the generalized counting rule of Ji et al. 23 to obtain the scaling behavior of the helicity flipping amplitudes.

It is well known that $p p$ scattering can be described by five independent helicity amplitudes [29]. According to the dimensional as well as the generalized counting rules the three helicity-conserving amplitudes, $M(+,+;+,+), M(+,-;+,-)$ and $M(-,+;+,-)$, have an energy dependence of $\sim 1 / s^{4}$. On the other hand the simple constituent quark interchange models [29] assume the two helicity flipping (nonconserving) amplitudes, $M(+,+;+,-)(N C 1)$ and $M(-,-;+,+)(N C 2)$ to be zero. Later analysis by Lepage and Brodsky [8] have shown these amplitudes to be non-zero but power suppressed. The new generalized counting rule predicts their energy dependence to be $\sim 1 / s^{4.5}$ and $\sim 1 / s^{5}$ respectively [23]. Thus the generalized counting, rule which includes the helicity flipping amplitudes and the interference between them, gives rise to additional energy dependence beyond the $s^{-10}$ scaling predicted by dimensional scaling.
In addition to these short distance amplitudes, Landshoff [30] has shown that there can be contributions from three successive on-shell quark-quark scattering. Although each scattering process is itself a short distance process, different independent scatterings can be far apart, limited only by the hadron size. The Landshoff amplitude also carries an energy dependent phase arising from gluonic radiative corrections which are calculable in pQCD [19] and has a known energy dependence, similar to the renormalization-group evolution: $\phi(s)=\frac{\pi}{0.06} \ln \ln \left(s / \Lambda_{Q C D}^{2}\right)$. This effect is believed to be analogous to the coulomb-nuclear interference that is observed in low-energy charged-particle scattering. It has been shown that this energy dependence of the phase occurs at medium energies 31] and becomes independent of energy at asymptotically high energies 31], 32]. In Ref. 18]; Ralston and Pire have used the helicityconserving amplitudes, the Landshoff amplitude with an energy dependent phase and the interference between them to reproduce the oscillations in the $p p$ scattering data at $90^{\circ}$ c.m. angle (a similar method was used by Carlson et. al 20] to describe oscillation in the crosssection as well as the spin-correlation). They write the two amplitudes as $M=M_{S}+e^{i \phi(s)+i \delta} M_{L}$, where $M_{S} \sim 1 / s^{4}$ represents the three helicity-conserving short distance amplitudes, $M_{L} \sim 1 / s^{3.5}$ is the Landshoff amplitude and $\phi(s)$ is the energy dependent phase, $\delta$ is an arbitrary energy independent phase. By fitting to the existing $p p$ scattering data at $90^{\circ} \mathrm{c} . \mathrm{m}$. angle, they find that the ratio of $M_{L}$ to $M_{S}$ is 1:0.04 for an energy dependent phase given by $\phi(s)=\frac{\pi}{0.06} \ln \ln \left(s / \Lambda_{Q C D}^{2}\right)$, where $\Lambda_{Q C D}$ $=100 \mathrm{MeV}$. It has been argued that the asymptotic leading limit used to calculate this energy dependence phase of the Landshoff amplitude is not entirely valid 33. and thus the Landshoff term is better parametrized as,

$$
M_{L}=b_{j} s^{-3.5} \frac{e^{i c_{j}\left[\ln \ln \left(s / \Lambda_{Q C D}\right)\right]+i \delta_{j}}}{[\log (s)]^{d_{j}}},
$$

where $b_{j}, c_{j}, d_{j}$ and the energy independent phase $\delta_{j}$ are now parameters which are not exactly calculable. Fig. [1, shows the fit of Ref. 18 compared to the world data, and Fig. 10 is a fit using the more general parametrization of the Landshoff described above. Both these fits deviate drastically from the data at $s<10 \mathrm{GeV}^{2}$ and are not sensitive to the different parameterizations of the Landshoff amplitude. Since the Landshoff amplitude is expected to be significant only at high energies, it is not unreasonable that the above formalism does not describe the data at low energies.

As the interference between the Landshoff and the short distance amplitudes fail to describe the data at low energies, it is possible that the helicity flip amplitudes and their interference may play an important role at these energies. The helicity flip amplitudes arising from the parton orbital angular momentum are non-negligible when the parton transverse momentum can not be ne- 


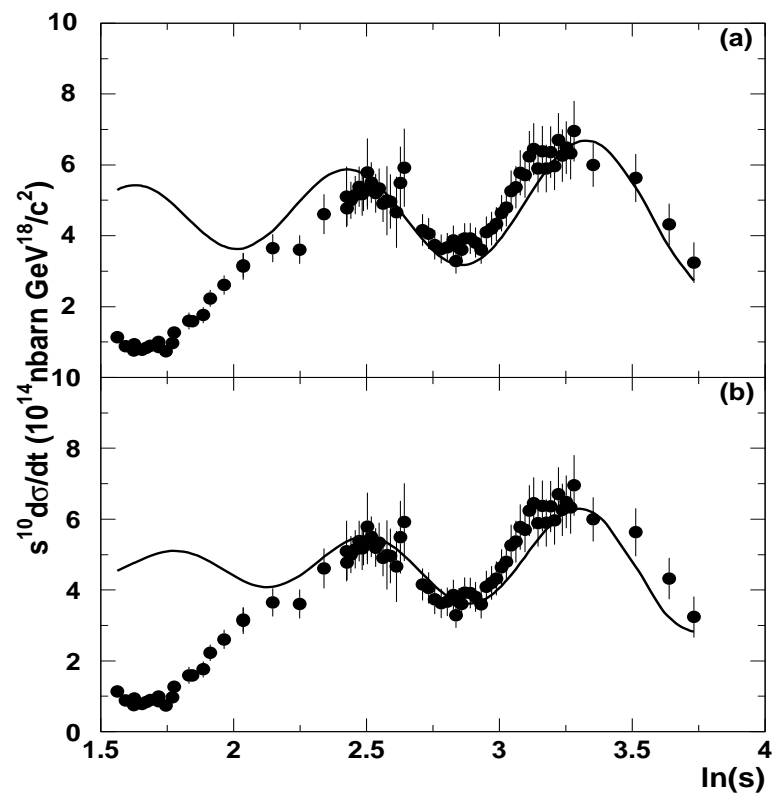

FIG. 1: (a) The fit to $p p$ scattering data at $\theta_{c m}=90^{\circ}$ of Ralston and Pire [18], this fit had two parameters; the overall normalization $A_{1}$ and the arbitrary phase $\delta$. (b) The same data fitted with the new more general parametrization of the Landshoff amplitude, this fit includes the 3 additional parameters $b_{1}, c_{1}$ and $d_{1}$ mentioned in Eq. 1 The data are from Ref. [12]

glected compared with the typical momentum scale in the exclusive processes at relatively low energies. Thus one would expect the helicity flip amplitudes to be a significant contribution to the cross-section at low energies. Moreover, the generalized counting rule of Ji et al. 23. predicts a much faster fall-off with energy for the helicity flip amplitudes as expected. We have refitted the world data by including the two helicity-nonconserving amplitudes according to the generalized counting rule of Ji et al. 23]. The two different forms for the energy dependence of the phase in the Landshoff amplitude, described above, were employed in the fits to examine their sensitivity to them. The three helicity-conserving amplitudes combined as one amplitude and the two helicity flipping amplitudes, along with the Landshoff contributions, can be written as;

$$
\begin{aligned}
M_{H C} & =s^{-4}\left(a_{1}+b_{1} s^{0.5} e^{i \phi_{1}(s)}\right) \\
M_{N C 1} & =s^{-4}\left(a_{2} s^{-0.5}+b_{2} s^{0.5} e^{i \phi_{2}(s)}\right) \\
M_{N C 2} & =s^{-4}\left(a_{3} s^{-1}+b_{3} s^{0.5} e^{i \phi_{3}(s)}\right),
\end{aligned}
$$

where $\phi_{j}(s)$ is the energy dependent phase. Two different forms for the phase $\phi_{j}(s)$ were used in our fits; $\phi_{j}(s)=$ $\frac{\pi}{0.06} \ln \ln \left(s / \Lambda_{Q C D}^{2}\right)+\delta_{j}$ and $\phi_{j}(s)=c_{j} \frac{\ln \ln \left(s / \Lambda_{Q C D}^{2}\right)+\delta_{j}}{(\log (s))^{d_{j}}}$. We have neglected the helicity flipping Landshoff contri- butions. The scaled cross-section is then given by,

$$
R=s^{10} \frac{d \sigma}{d t} \propto\left|M_{H C}\right|^{2}+4\left|M_{N C 1}\right|^{2}+\left.M_{N C 2}\right|^{2},
$$

The factor of four associated with the $N C 1$ helicity flipping amplitude arises because of the two possible configurations of this single spin flip amplitude [29].

Fig 2 shows the results of our fit and also shows the explicit contributions from the $s^{-11}$ and $s^{-12}$ term for this approach. The value of $\Lambda_{Q C D}$ was fixed at $100 \mathrm{MeV}$ for all fits. This new fit is in much better agreement with the data. The helicity flip amplitudes (mostly the term $\sim s^{-4.5}$ ) are significant at low energies and seem to help in describing the data at low energies. It is interesting to note that among the helicity flip amplitudes the one with the lower angular momentum dominates. These are very promising results and should be examined for other reactions.

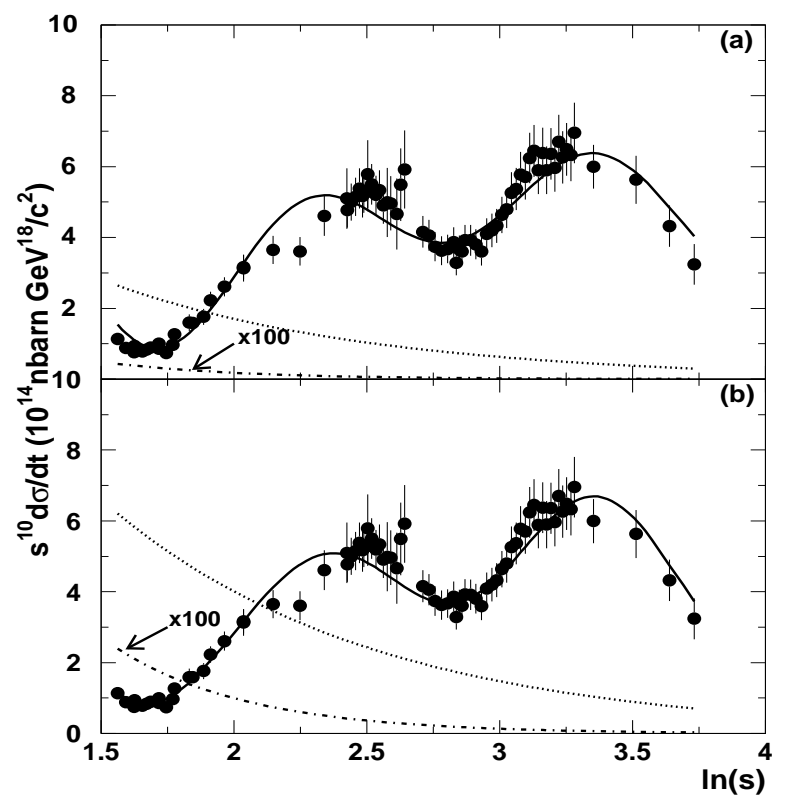

FIG. 2: (a) The fit to $p p$ scattering data at $\theta_{c m}=90^{\circ}$ when helicity flip amplitudes are included as described in Eq. 2] The parameters for the energy dependent phase was kept same as the earlier fit of Ralston and Pire [18]. The solid line is the fit result, the dotted line is contribution from the helicity flip term $\sim s^{-11}$, the dot-dashed line is contribution from the helicity flip term $\sim s^{-12}$. The $\sim s^{-12}$ contribution has been multiplied by 100 for display purposes.(b)The same data fitted to the form described in Eq. 2 but with the new more general parametrization of the Landshoff amplitude which includes the 3 additional parameters per term, $b_{j}, c_{j}$ and $d_{j}$ $(j=1,2,3)$ as mentioned in Eq. 1]

As mentioned earlier the $A_{N N}$ spin-correlation in polarized $p p$ elastic scattering also shows large deviations [16] from the expectations of pQCD (assuming 
hadron helicity is conserved). In terms of the helicity amplitudes $A_{N N}$ is given by [29];

$$
\begin{aligned}
R A_{N N} & =2 \operatorname{Re}\left[M^{*}(++;++) M(--;++)\right] \\
& +2 \operatorname{Re}\left[M^{*}(+-;+-) M(-+;+-)\right] \\
& +4|M(++;+-)|^{2},
\end{aligned}
$$

where $R$ has been defined in Eq. 3. At $\theta_{c m}=90^{\circ}$ the ratio of the three helicity non-flip amplitudes is $2: 1: 1$ [29]. Taking this into account we have fit the $A_{N N}$ data by including the helicity flipping amplitudes. Fig. 3 a shows the results for the case where the helicity flip amplitude is neglected and only the interference between short distance amplitude and the Landshoff amplitude is used (in this case the expression for $A_{N N}$ simplifies to $\left.R A_{N N}=2 \operatorname{Re}\left[M^{*}(+-;+-) M(-+;+-)\right]\right)$. These results are similar to those obtained by Carlson et. al [20] and they described the $A_{N N}$ data at high energies but fail to describe the low energy data using this idea of interference between short distance and Landshoff terms. Fig. 3b shows the results of our fit when the helicity flipping amplitudes are included. It is clear that this method is a better fit to a larger fraction of the data which includes some low energy data. This suggests that even in case of the spin correlation $A_{N N}$ in polarized $p p$ elastic scattering the helicity flip amplitudes play an important role at low energies $\left(s<10 \mathrm{GeV}^{2}\right)$.

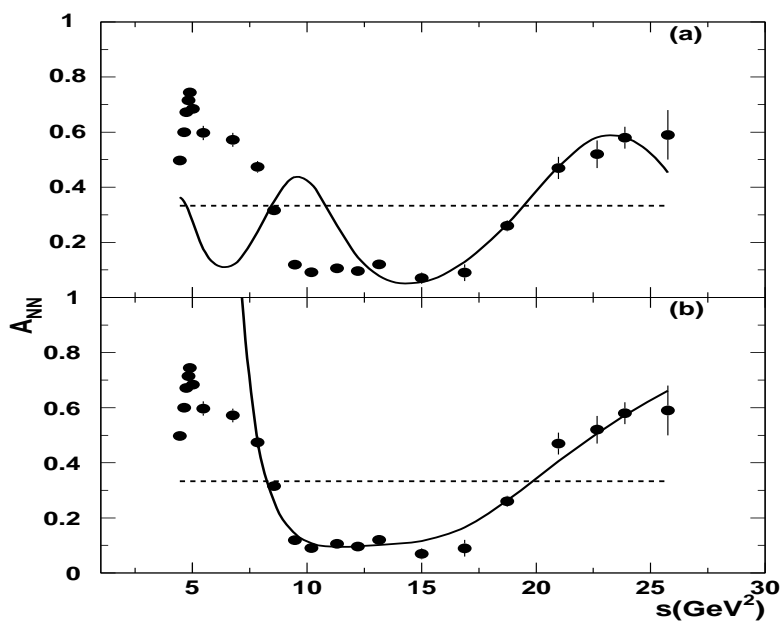

FIG. 3: (a) The fit to $A_{N N}$ from polarized $p p$ scattering data at $\theta_{c m}=90^{\circ}$ with the helicity non-flip and Landshoff amplitudes only. (b) Fit to the same data when the helicity flip amplitudes are included. The data are from Ref. [15, 16]. The solid line is the fit and the dashed line is the expectation assuming hadron helicity conservation.

Recently some precision data on pion-photoproduction from nucleons above the resonance region has become available from JLab [5]. These data show hints of oscillation about the $s^{-7}$ scaling predicted by the quark count- ing rule. In pion-photoproduction from nucleons the helicity non-flip amplitudes has an energy dependence of $s^{-2.5}$, and there is just one helicity flip amplitude which according to the generalized counting rule has an energy dependence of $s^{-3}$ 23]. There are no leading order Landshoff terms in pion-photoproduction since the initial state has a single hadron. However, the Landshoff process can contribute at sub-leading order 34] (i.e. $\sim s^{-3}$ instead of $\sim s^{-2}$ ). In principle, the fluctuation of a photon into a $q \bar{q}$ in the initial state can contribute an independent scattering amplitude at sub-leading order. But, experimentally it has been shown that vector-meson dominance diffractive mechanism is suppressed in vector meson photoproduction at large values of $t$ [35]. On the other hand such independent scattering amplitude can contribute in the final state if more than one hadron exist in the final state, as is the case in nucleon photo-pion production reactions. Thus an unambiguous confirmation of such an oscillatory behavior in exclusive photoreactions with hadrons in the final state at large $t$ may provide a signature of QCD final state interaction.

We have fit the pion-photoproduction data at $\theta_{c m}=$ $90^{\circ}$ including the helicity flip amplitude and the Landshoff amplitude at sub leading order with an energy dependent phase. The Landshoff amplitude was parametrized according to the ansatz given in Ref. [33]. The amplitudes for $\gamma p \rightarrow \pi^{+} n$ and $\gamma n \rightarrow \pi^{-} p$ and the respective Landshoff contribution to each amplitude can be written as;

$$
\begin{aligned}
M_{H C} & =s^{-2.5}\left(a_{1}+b_{1} s^{-0.5} \frac{e^{i c_{1} \phi(s)+i \delta_{1}}}{(\log (s))^{d_{1}}}\right) \\
M_{N C 1} & =s^{-2.5}\left(a_{2} s^{-0.5}+b_{2} s^{-0.5} \frac{e^{i c_{2} \phi(s)+i \delta_{2}}}{(\log (s))^{d_{2}}}\right),
\end{aligned}
$$

and the scaled cross-section is given by; $s^{7} \frac{d \sigma}{d t} \propto\left|M_{H C}\right|^{2}+\left|M_{N C 1}\right|^{2}$, where $\phi(s)=\ln \ln \left(s / \Lambda^{2}\right)$. As seen in Fig 4 the existing data can be fit quite well with this form. However, the data are rather coarsely distributed in energy and so these results are not a conclusive evidence for oscillations in pion-photoproduction. This underscores the point that a fine scan of energies above the resonance region is urgently needed. This is exactly the issue that will be addressed in the JLab experiment E02010 36] in the near future.

We have shown that the generalized counting rule of Ji et al. 23] along with the Landshoff terms and associated interferences does a better job of describing the oscillations about the quark counting rule, in the $p p$ elastic scattering data at $\theta_{c m}=90^{\circ}$. This is specially true in the low energy region $\left(s<10 \mathrm{GeV}^{2}\right)$. The contributions from helicity flipping amplitudes which are related to quark orbital angular momentum, seem to play an important role at these low energies, which is reasonable given that the quark orbital angular momentum is non-negligible compared to the momentum scale of the 


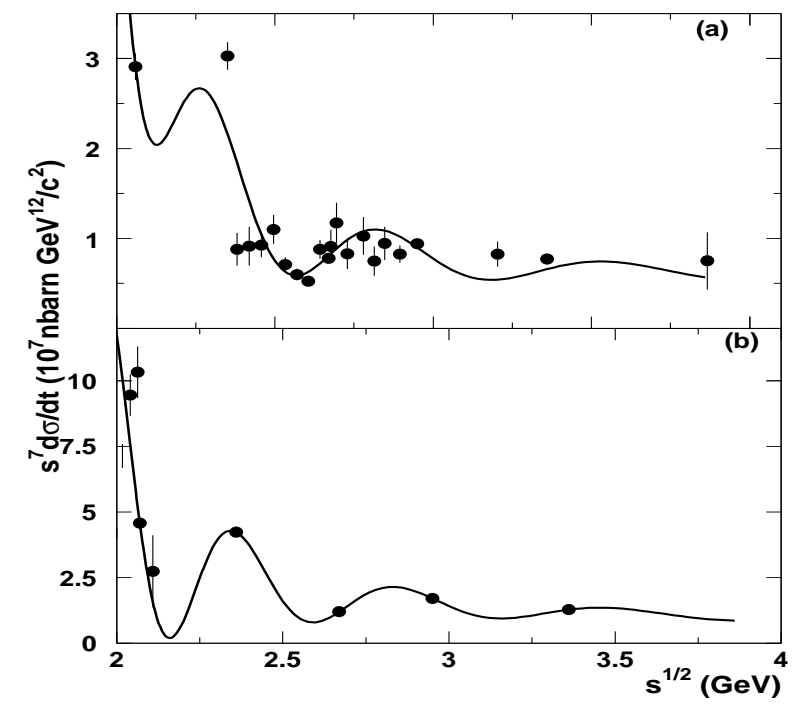

FIG. 4: (a) The fit to $\gamma p \rightarrow \pi^{+} n$ scattering data at $\theta_{c m}=90^{\circ}$ when helicity flip and sub-leading order Landshoff amplitudes are included (b) Fit to $\gamma n \rightarrow \pi^{-} p$ scattering data at $\theta_{c m}=$ $90^{\circ}$. The data are from Ref. [5, 14].

scattering process. Similarly the spin-correlation $A_{N N}$ in polarized $p p$ elastic scattering data can be better described by including the helicity flipping amplitude along with the Landshoff amplitude and their interference. The photo-pion production data from nucleons at large angles can also be described similarly; however, because of the coarse energy spacing of the data, the results are not as illustrative. This points to the urgent need for more data on pion-photoproduction above the resonance region with finer energy spacing. We expect that our experiment at JLab which is approved for running will help address this need in the near future.

We acknowledge fruitful discussions with X. Ji and S. J. Brodsky. This work is supported by the U.S. Department of Energy under contract number DE-FG0203ER41231.

[1] C. White et al., Phys. Rev. D49, 58 (1994).

[2] S. J. Brodsky and G.R. Farrar, Phys. Rev. Lett.31, 1153 (1973); Phys. Rev. D 11, 1309 (1975); V. Matveev et al., Nuovo Cimento Lett. 7, 719 (1973);

[3] C. Bochna et al., Phys. Rev. Lett. 81, 4576 (1998); E.C. Schulte, et al., Phys. Rev. Lett. 87, 102302 (2001);

[4] P. Rossi et al., Phys. Rev. Lett 94, 012301 (2005); M. Mirazita et al., Phys. Rev. C 70, 014005 (2004).

[5] L. Y. Zhu et al., Phys. Rev. Lett. 91, 022003 (2003); L. Y. Zhu et al., nucl-ex/0409018

[6] S. J. Brodsky and G. P. Lepage, Phys. Rev. D 24, 2848 (1981).
[7] K. Wijesooriya, et al., Phys. Rev. Lett. 86, , (2)975 (2001).

[8] G. P. Lepage, and S. J. Brodsky, Phys. Rev. D 22, 2157 (1980).

[9] T. Gousset, B. Pire and J. P. Ralston, Phys. Rev. D 53, 1202 (1996).

[10] J. P. Ralston and P. Jain, Phys. Rev. D 69, 053008 (2004).

[11] N. Isgur and C. H. Llewellyn Smith, Phys. Rev. Lett. 52, 1080 (1984).

[12] C. W. Akerlof, et al., Phys. Rev. 159, 1138 (1967); R. C. Kammerud, et al., Phys Rev. D 4, 1309 (1971); K. A. Jenkins, et al., Phys. Rev. Lett, 40, 425 (1978).

[13] A.W. Hendry, Phys. Rev. D 10, 2300 (1974).

[14] D. P. Owen et al., Phys. Rev. 181, 1794 (1969); K. A. Jenkins et al., Phys. Rev. D 21, 2445 (1980); C. Haglin et al., Nucl. Phys. B 216, 1 (1983).

[15] D.G. Crabb et al., Phys. Rev. Lett. 41, 1257 (1978).

[16] G.R. Court et al., Phys. Rev. Lett. 57, 507 (1986);T.S. Bhatia et al., Phys. Rev. Lett. 49, 1135 (1982); E.A. Crosbie et al., Phys. Rev. D 23, 600 (1981).

[17] S. J. Brodsky, and G. F. deTeramond; Phys. Rev. Lett. 60, 1924 (1988).

[18] J. P. Ralston and B. Pire, Phys Rev. Lett. 49, 1605 (1982); B. Pire and J. P. Ralston, Phys. Lett. B 117, 233 (1982)

[19] A. Sen, Phys. Rev. D 28, 860 (1983).

[20] C.E. Carlson, M. Chachkhunashvili, and F. Myhrer, Phys. Rev. D 46, 2891 (1992).

[21] L.L. Frankfurt, G.A. Miller, M.M. Sargsian, and M.I. Strikman, Phys. Rev. Lett. 84, 3045 (2000).

[22] Q. Zhao and F. E. Close, Phys. Rev. Lett. 91, 022004 (2003).

[23] X. Ji, J.-P. Ma and F. Yuan, Phys. Rev. Lett. 90, 241601 (2003).

[24] J. Polchinski and M.J. Strassler, Phys. Rev. Lett. 88, 031601 (2002); R.C. Brower and C.I. Tan, Nucl. Phys. B 662, 393 (2003); O. Andreev, Phys. Rev. D 67, 046001 (2003).

[25] S. J. Brodsky and G. F. de Teramond, Phys. Lett. B582, 211 (2004); S. J. Brodsky,J. R. Hiller, D. S. Hwang and V. A. Karmanov, Phys. Rev. D 69, 076001 (2004).

[26] R. Buniy, J. P. Ralston and P. Jain in VII International Conference on the Intersections of Particle and Nuclear Physics, Quebec City, 2000, edited by Z. Parsa and W. Marciano (AIP, New York, 2000), hep/ph/0206074

[27] A. V. Belitsky, X. Ji and F. Yuan, Phys. Rev. Lett. 91, 092003 (2003).

[28] M. K. Jones et al., Phys. Rev. Lett. 84, 1398 (2000); O. Gayou et al., Phys. Rev. Lett. 88, 092301 (2002).

[29] S. J. Brodsky, C. E. Carlson and H. Lipkin, Phys. Rev. D 20, 2278, (1979);G. R. Farrar, S. Gottlieb, D. Sivers and G. H. Thomas, Phys. Rev. D 20, 202 (1979).

[30] P. V. Landshoff, Phys. Rev. D 10, 1024 (1974).

[31] J. Botts and G. Sterman, Nucl. Phys. B325, 62 (1989).

[32] A. H. Mueller, Phys. Rep. 73, 237 (1981).

[33] P. Jain, B. Kundu and J. P. Ralston, Phys. Rev. D 65, 094027 (2002).

[34] G.R. Farrar, G. Sterman, and H. Zhang, Phys. Rev. Lett. 62, 2229 (1989).

[35] E. Anciant et al., Phys. Rev. Lett. 85, 4682 (2000).

[36] JLab experiment E02-010, Spokespersons D. Dutta, H. Gao and R. Holt (2002). 